\title{
Facile Synthesis of Formate-Functionalized Graphene Quantum Dots
}

\author{
Jonathan A. Bruce* and Jude C. Clapper \\ Taipei American School, 800 Chung Shan North Road, Section 6, Taipei, Taiwan 11152
}

\begin{abstract}
Graphene quantum dots have proven useful for a variety of applications due to their impressive bandgap tunability, which can be achieved through structural modification methods including edge functionalization and doping. Here, we investigate the functionalization of graphene quantum dots with formate esters using a method of noncatalyzed electrophilic substitution. We also examine the optical and structural properties of these formate-functionalized graphene quantum dots using UV-Vis Spectrophotometry, IR Spectroscopy, and NMR Spectroscopy, with the functionalization causing a redshift in absorption consistent with a reduction in bandgap. The findings in this paper have the potential to be used in applications ranging from single photon emission in quantum computing to tunable fluorescence in bioimaging.
\end{abstract}

\section{INTRODUCTION}

Graphene, an allotrope of carbon, has garnered much attention since its experimental discovery in 2004 due to its unique properties. ${ }^{1}$ Being one atom thick and containing a hexagonal, $\mathrm{sp}^{2}$-hybridized lattice-type structure, graphene possesses a low mass coupled with impressive structural strength. ${ }^{2}$ Additionally, graphene's electronic band structure allows for high electron mobility, making it an excellent conductor of heat and electricity. ${ }^{3}$ Finally, graphene - even in quantum dot form - poses low health risks, ${ }^{4}$ and is easily obtainable from graphite, a common material composed of stacked sheets of graphene. This combination of ideal properties permits many applications of graphene in electronics, bioimaging, biosensing, and more.

In recent years, a new application of graphene has received attention - graphene quantum dots (GQDs), or 0-dimensional particles of graphene. ${ }^{5}$ GQDs inherit many of graphene's desirable physical properties, such as high electron mobility and low toxicity. Additionally, due to their size (on the order of $10^{-8} \mathrm{~m}$ ), GQDs are also subject to quantum confinement, and exhibit tunable photoluminescence as a result. ${ }^{6}$ Due to these ideal properties, GQDs have found a home in a variety of applications, including solar cells, ${ }^{7}$ lithium-ion batteries, ${ }^{8}$ bioimaging, ${ }^{9}$ and single-photon emission. ${ }^{10}$
As with other types of quantum dots, such as those composed of amorphous carbon, there exists the potential to enhance the photoluminescence (PL) properties of GQDs through modification of their chemical structures. One common method of doing so is the introduction of a dopant into the structure of a GQD - for instance, Ain et al. have examined the effects of doping GQDs with boron, nitrogen, chlorine, sodium, and potassium, and have found significant differences in PL properties following the introduction of the dopants. ${ }^{11}$ Another avenue for altering PL characteristics, which forms the focus of this paper, involves functionalizing the edge structures of graphene quantum dots. Multiple approaches to edge functionalization have been examined in the past: Kumar et al., among others, have examined aminofunctionalized graphene oxide QDs, ${ }^{12}$ and $\mathrm{Li}$ et al. have performed a theoretical study on GQDs functionalized with a variety of chemical groups, many containing $\mathrm{C}=\mathrm{O}$ double bonds. ${ }^{13}$ However, there still exist a variety of potential candidates for edge functionalization that have yet to be explored. As such, the focus of this paper is to create and experimentally verify a protocol for the functionalization of GQDs using formate, which, to our best knowledge, has yet to be experimentally achieved. We also aim to examine the effect of formate functionalization on the optical absorbance properties of GQDs. 


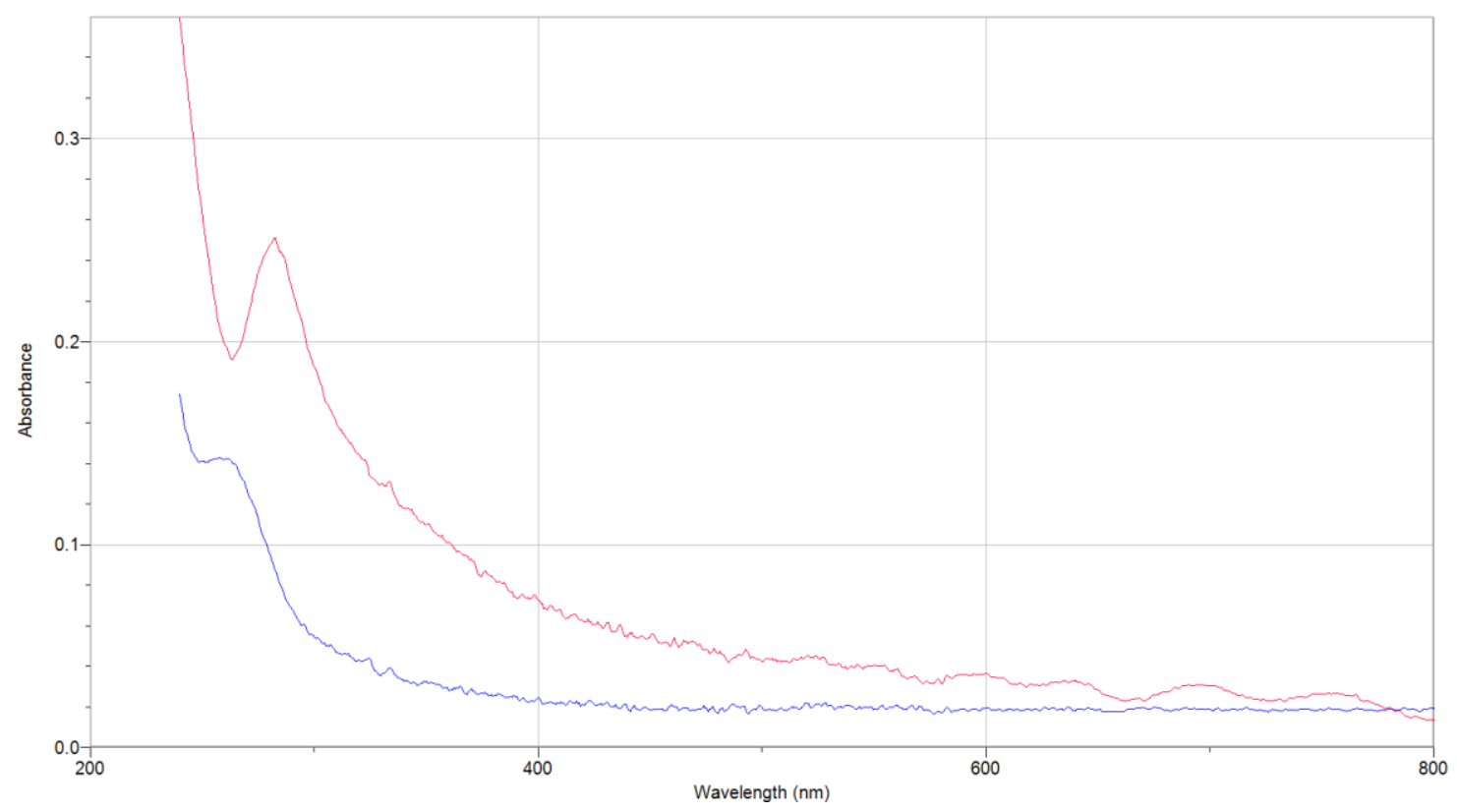

Figure 1: Image of the UV-Vis spectrum for GQDs (blue curve) and functionalized GQDs (red curve), showing a redshift in absorption due to a reduction in bandgap as a result of functionalization. Different concentrations of functionalized/non-functionalized quantum dots were used.

\section{EXPERIMENTAL METHODS}

\section{Graphene Quantum Dot Fabrication}

To fabricate non-functionalized GQDs, an electrochemical process derived from the work of Ahirwar et al. was used. ${ }^{14}$ To start, $500 \mathrm{~mL}$ of a solution containing $0.10 \mathrm{M}$ citric acid monohydrate (Showa Chemical) and $0.30 \mathrm{M}$ sodium hydroxide (Choneye Pure Chemicals), dissolved in Milli-Q water, was prepared. This 1:3 molar ratio of citric acid to $\mathrm{NaOH}$ was chosen for its ability to produce GQDs with low amounts of oxygen-containing functional groups, in addition to high amounts of $\mathrm{C}-\mathrm{H}$ bonds on the GQDs' surface. ${ }^{14}$ Two graphite rods were then heated at $\sim 1000^{\circ} \mathrm{C}$ for $\sim 10$ minutes to introduce surface impurities before being rinsed in Milli-Q water, dried, and placed vertically in the solution $25 \mathrm{~mm}$ apart.

Using a DC power supply, a voltage was passed through the rods for 15 minutes, alternating between +0.5 and $+1.2 \mathrm{~V}$ every $\sim 5$ seconds with current between 0.005 and $0.010 \mathrm{~A}$. Following this, a constant voltage of $+10.0 \mathrm{~V}$ (with current equal to $0.200 \mathrm{~A}$ ) was applied to the rods for 30 minutes. The solution gradually turned from clear to yellow, indicating the formation of GQDs. $0.15 \mathrm{M}$ calcium chloride was then added to the solution and heated to $70^{\circ} \mathrm{C}$ for 15 minutes to precipitate calcium citrate. The precipitate was filtered out of solution, and the solution was placed in dialysis tubing (\#684216, Carolina) for 7 days.

After 7 days, a yellow-colored solid was obtained after the removal of water via rotary evaporation. UV-Vis spectrophotometry (VSPUV, Vernier), IR spectroscopy (UATR Two, PerkinElmer), and ${ }^{1} \mathrm{H}$ NMR spectroscopy were performed to characterize the sample. Prior to ${ }^{1} \mathrm{H}$ NMR spectroscopy (Avance, Bruker), the sample was dispersed in ethanol to isolate GQDs from any remaining calcium citrate from the synthesis process, and then reduced to dryness and rehydrated in $\mathrm{D}_{2} \mathrm{O}$.

\section{Graphene Quantum Dot Functionalization with Formate}

To functionalize the GQD sample with formate, an excess of formic acid (determined using 1:1 molar ratio based on total GQD mass 
and molar mass of carbon) was added to the sample in a flask.

The resulting solution was refluxed and stirred for 48 hours, at $95^{\circ} \mathrm{C}$, inside of a fume hood. For UV-Vis spectrophotometry (VSP-UV, Vernier) and IR spectroscopy (UATR Two, PerkinElmer), this functionalized GQD sample was placed in dialysis tubing (\#684216, Carolina) for 7 days to filter any excess formic acid. For ${ }^{1} \mathrm{H}$ NMR spectroscopy (Avance, Bruker), this functionalized GQD sample was dispersed in ethanol to isolate GQDs from any remaining calcium citrate from the synthesis process, and then reduced to dryness and rehydrated in $\mathrm{D}_{2} \mathrm{O}$.

\section{RESULTS AND DATA}

\section{UV-Vis Spectrophotometry}

Results of the formate-functionalized GQDs demonstrate marked differences compared to non-functionalized GQDs. UV-Vis spectrophotometry demonstrates a prominent shift in absorbance, from a peak at $255 \mathrm{~nm}$ for non-functionalized GQDs to a peak at $282 \mathrm{~nm}$ for functionalized GQDs (Figure 1). This redshift in absorbance demonstrates a decreased bandgap width in the formate-functionalized GQD as compared to the non-functionalized GQD. This is consistent with the theoretical results of $\mathrm{Li} e t$ $a l$. for other functional groups - namely, a longer wavelength of light implies a lower energy of emitted light and therefore a smaller bandgap. ${ }^{13}$

\section{IR-Spectroscopy Results}

The IR spectrum of the non-functionalized GQDs shows a well-defined peak at $1591 \mathrm{~cm}^{-1}$ corresponding to hybridized carbon-carbon bonds in the structure of graphene. ${ }^{15}$ Additionally, peaks at 2924 and $2854 \mathrm{~cm}^{-1}$ correspond to $\mathrm{C}-\mathrm{H}$ bonds found in the edge structure of graphene, which appear in prior literature at 2922 and $2850 \mathrm{~cm}^{-1} .{ }^{15}$ Additional peaks at $\sim 1700, \sim 1400$, and $\sim 1250 \mathrm{~cm}^{-1}$ are thought to be due to residual impurities, especially citric acid, from the synthesis procedure (Figure 2a). ${ }^{16}$
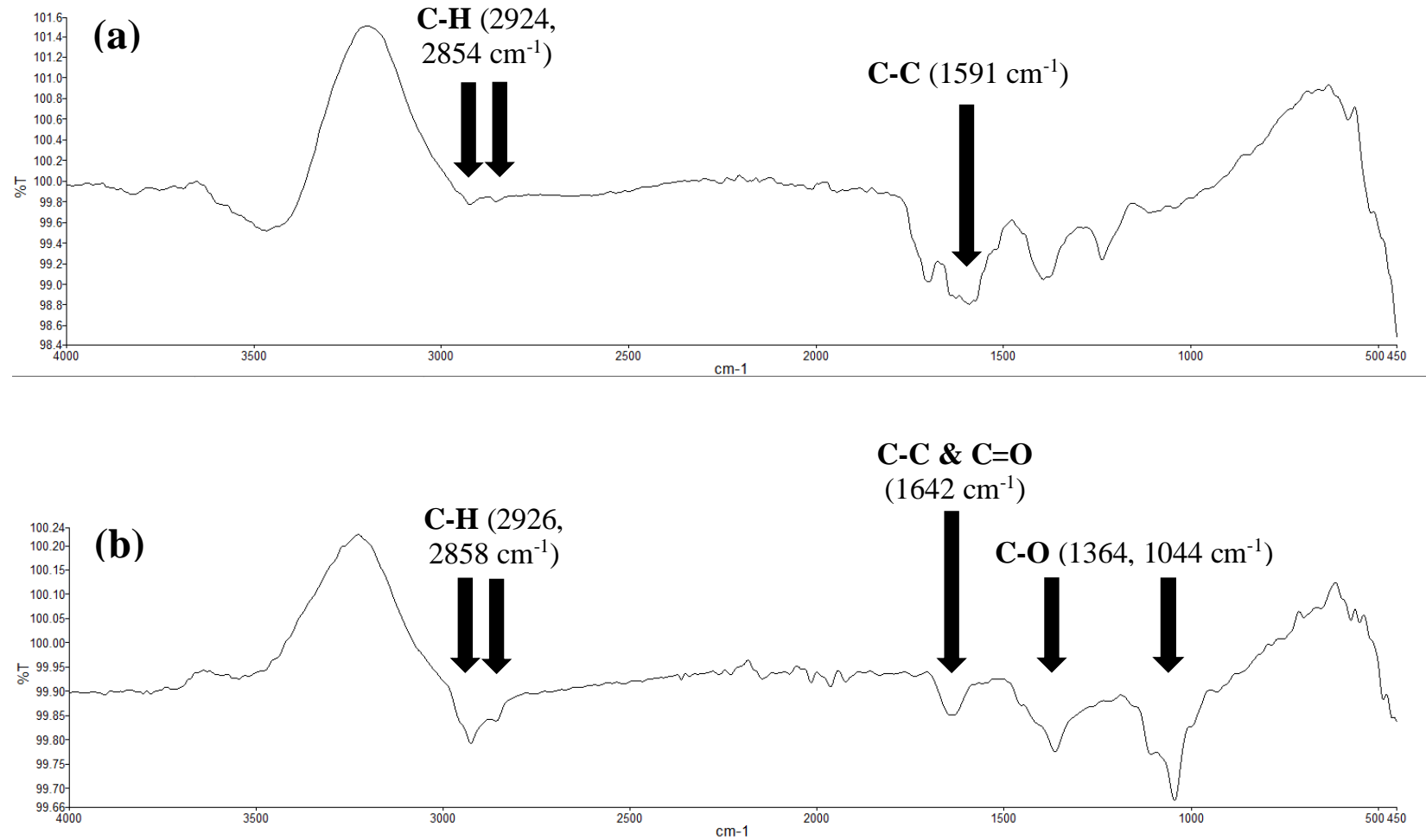

Figure 2: Image of the IR-spectroscopy graph for the (a) non-functionalized and (b) formate-functionalized GQD samples, with the peaks of interest highlighted and labeled. Different concentrations of functionalized/nonfunctionalized quantum dots were used. 

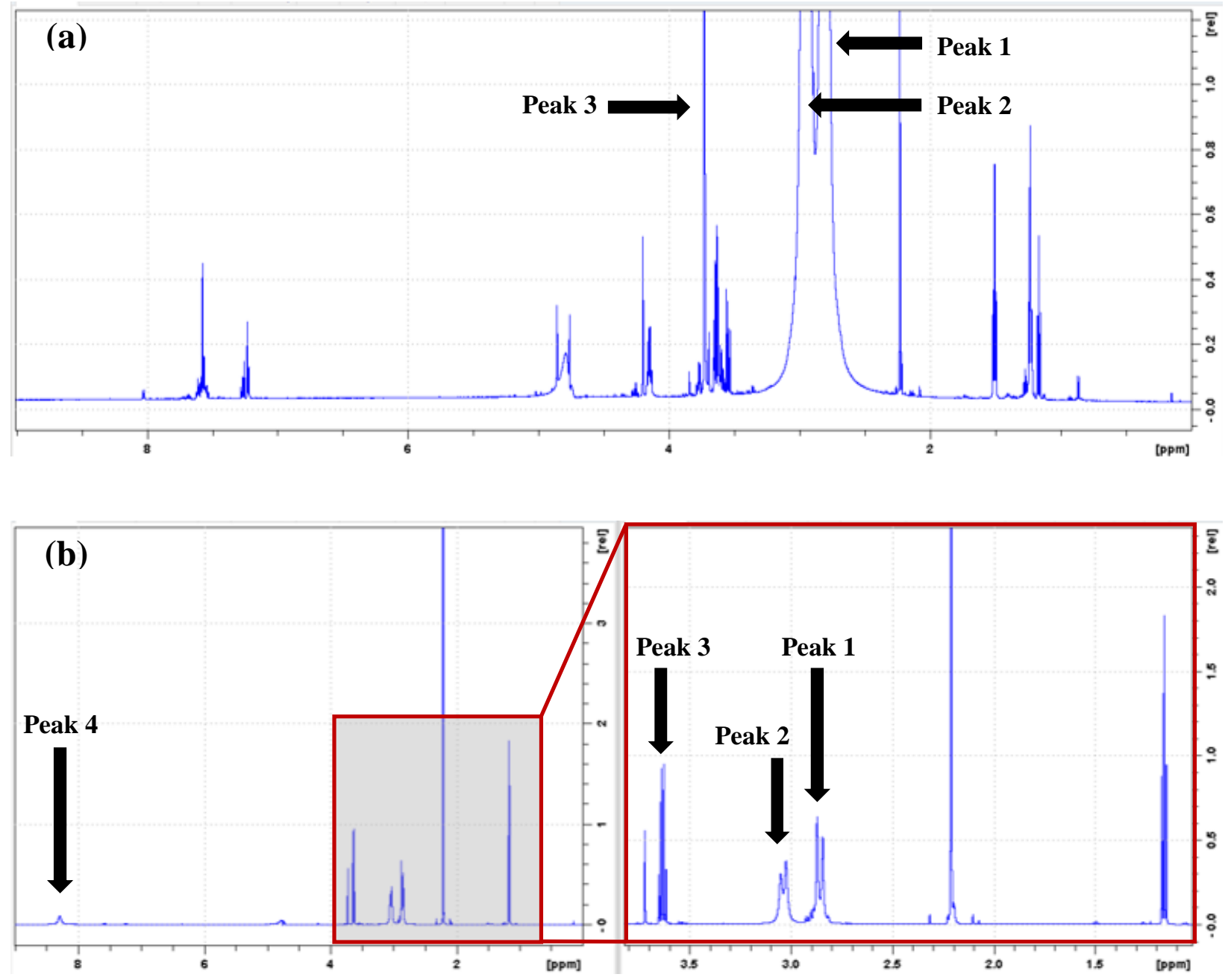

Figure 3: Images of the ${ }^{1} \mathrm{H}$ NMR spectra for (a) GQDs and (b) functionalized GQDs. Peaks of interest in each graph are labelled, and the shaded portion of spectrum (b) was enlarged for clarity. Note: Samples were of different concentrations (approximately $5 \mathrm{mg} / \mathrm{mL}$ ).

The IR spectrum of the formatefunctionalized GQD sample shows a pair of small peaks at 2926 and $2858 \mathrm{~cm}^{-1}$, two broad peaks located at 1642 and $1364 \mathrm{~cm}^{-1}$, and a sharp peak at $1044 \mathrm{~cm}^{-1}$ (Figure 2b).

The broad peak at $1642 \mathrm{~cm}^{-1}$ is attributed to the hybridized carbon-carbon bonds inherent in graphene's structure, ${ }^{15}$ as well as the $\mathrm{C}=\mathrm{O}$ bonds inherent in formate. ${ }^{17}$ Likewise, the two peaks at 2926 and $2858 \mathrm{~cm}^{-1}$ correspond to $\mathrm{C}-\mathrm{H}$ bonds in the GQDs. ${ }^{15}$

Finally, the peaks located at 1364 and 1044 $\mathrm{cm}^{-1}$, after comparison with the IR spectra for pure formic acid and graphene oxide, ${ }^{15,17}$ are associated with $\mathrm{C}-\mathrm{O}$ bonds.

\section{NMR Spectroscopy}

Additionally, ${ }^{1} \mathrm{H}$ NMR spectroscopy was performed to characterize the GQDs' structure (Figure 3).

Comparison with NMR spectra for formic acid suggests that peak 4 in the functionalized GQD spectrum (Figure 3b), which does not appear in the non-functionalized GQD spectrum, corresponds to the formate functional group. ${ }^{18}$

NMR spectra for GQDs suggests that peaks 1, 2, and 3 in both Figures $\mathbf{3 a}$ and $\mathbf{3 b}$ correspond to the structure of a non-functionalized GQD, independent of any functional groups. ${ }^{19}$ 
The additional peaks in both samples correspond to residual ethanol and $\mathrm{H}_{2} \mathrm{O}$ from the purification process.

\section{FURTHER ANALYSIS}

The edge-functionalization protocol detailed here is derived from the process of electrophilic substitution, in which one electrophile replaces another in the edge structure of a GQD. Therefore, formate, an electrophile, will tend to replace hydrogen atoms in the edge structure of a GQD through electrophilic substitution, thus functionalizing the GQD (Figure 4).
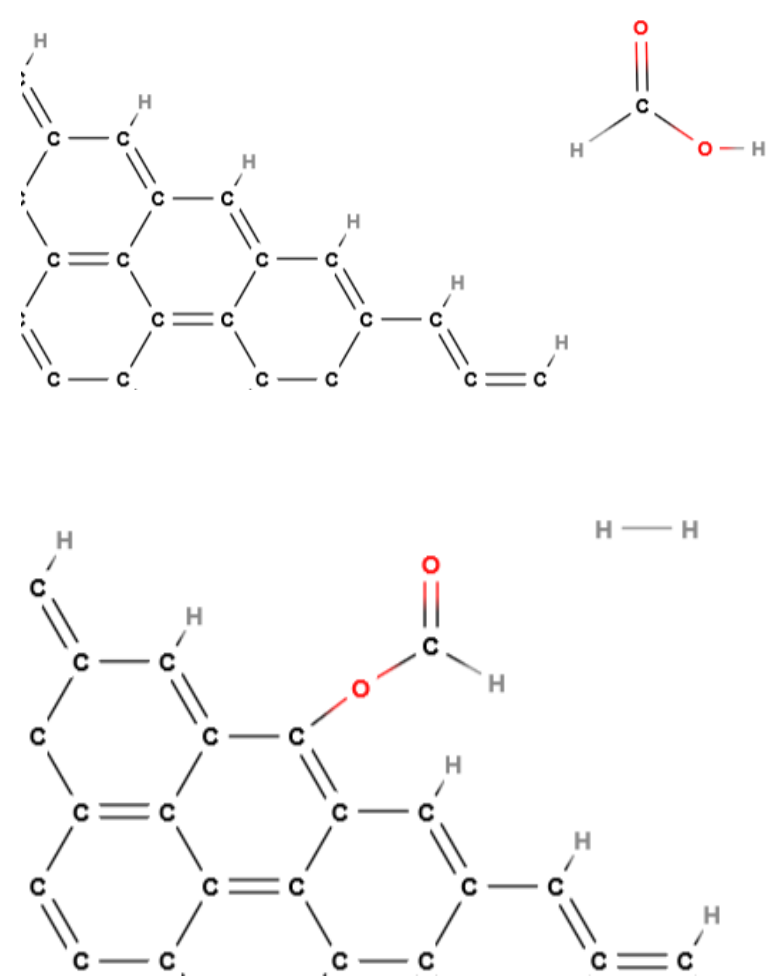

Figure 4: Diagram of proposed mechanism, before (top) and after (bottom) GQD functionalization with formate.

As a part of this functionalization mechanism, the edge structures of nonfunctionalized GQDs are assumed to contain abundant $\mathrm{C}-\mathrm{H}$ bonds. Typically, top-down synthesis methods yield significant oxygencontaining functional groups on the surface of GQDs, such as -OH and -COOH. ${ }^{20}$ However, for
GQDs synthesized using a 1:3 molar ratio of citric acid to $\mathrm{NaOH}$, the XPS data of Ahirwar et al. show an abundance of C-H bonds (by At. \%) in the GQDs' surface structures. Furthermore, the XPS data also show that these $\mathrm{C}-\mathrm{H}$ bonds are significantly greater in amount than $\mathrm{C}-\mathrm{O}$ and $\mathrm{C}=\mathrm{O}$ bonds, which represent oxygen-containing functional groups. ${ }^{14}$ Thus, Figure 4 depicts edges entirely containing $\mathrm{C}-\mathrm{H}$ bonds for illustrational purposes only; our GQDs could contain low amounts of other functional groups.

Evidence of the functionalized GQD structure, shown in Figure 4, can be found in the IR spectra. Specifically, the peak at $1364 \mathrm{~cm}^{-1}$ in Figure $\mathbf{2 b}$ is attributed to the $\mathrm{C}-\mathrm{O}$ bond in the formate functional group. This peak appears, with a $\sim 150 \mathrm{~cm}^{-1}$ shift, in the IR spectrum for pure formic acid. ${ }^{17}$ The observed shift can be attributed to the change from a $\mathrm{C}-\mathrm{OH}$ bond to a $\mathrm{C}-\mathrm{O}-\mathrm{C}$ bond when the formate ion attaches to the GQD. Meanwhile, the peak at $1044 \mathrm{~cm}^{-1}$ in Figure 2b aligns closely with the $1084 \mathrm{~cm}^{-1} \mathrm{C}-\mathrm{O}$ peak observed in the IR spectrum for graphene oxide, ${ }^{15}$ a compound that has oxygen bonded to graphene in a similar way as in our proposed structure.

Additionally, the $\mathrm{C}-\mathrm{O}$ peaks at $1364 \mathrm{~cm}^{-1}$ and $1044 \mathrm{~cm}^{-1}$ seen in Figure $\mathbf{2 b}$ are not observed in Figure 2a, indicating that $\mathrm{C}-\mathrm{O}$ bonds were formed during functionalization. Therefore, the C-O peaks observed in the spectrum for functionalized GQDs reinforce our predicted structure.

Further evidence of this proposed structure can be found in peak 4 of the ${ }^{1} \mathrm{H}$ NMR spectrum for functionalized GQDs (Figure 3b), which shows a slight shift to $\sim 8.3 \mathrm{ppm}$ from the peak at $8.44 \mathrm{ppm}$ associated with formic acid. ${ }^{18}$ This shift was attributed to the change from a $\mathrm{C}-\mathrm{OH}$ bond in formic acid to a $\mathrm{C}-\mathrm{O}-\mathrm{C}$ bond linking the formate ester to the GQD. Therefore, the NMR spectra indicate the presence of formate functional groups in the edge structures of functionalized GQDs, as predicted.

\section{CONCLUSION}

Through a facile experimental protocol, we have successfully executed edge 
functionalization of GQDs with formate. Furthermore, we have used IR Spectroscopy and NMR Spectroscopy to analyze the structural properties of our functionalized GQDs and have found that they support our theoretical structure. Finally, we have analyzed the absorbance properties of these GQDs and confirmed a redshift in absorption, characteristic of a reduction in bandgap.

These findings hold the potential for several real-world applications, including bioimaging and single photon emission in quantum computing. These applications require the precise bandgap tunability and control over optical properties observed in this study. Furthermore, owing to the low toxicity of GQDs and the relatively low toxicity of formate, we predict acceptably low toxicity levels for use in such applications, especially compared to GQDs functionalized with other groups such as halogens.

\section{ACKNOWLEDGEMENTS}

The authors are grateful to Dr. Theresa W. Lynn, Physics Department Chair, Harvey Mudd College, for consultation on edge functionalization of nanoparticles and single photon emission. The authors thank Dr. Dennis W. Hwang, Dr. Vivian Chen, and Dr. Yuan-Chao Lou of Academia Sinica, Institute of Biomedical Sciences, for consultation and NMR Spectroscopy. The authors thank Dr. Michael Li and Mr. Yi-Ta Hsieh of National Yang Ming University, School of Dentistry, for UV-Vis Spectrophotometry and NMR Spectroscopy. The authors thank Dr. Allan Bayntun of Taipei American School for advice on exfoliation of graphite rods, Dr. Nicholas Ward of Taipei American School for consultation on single photon emission, and Mr. Sean Tsao of Taipei American School for resources.

\section{AUTHOR CONTRIBUTIONS}

JAB: Conceptualization, Methodology \& Data Curation, Investigation, Validation, Formal Analysis, Visualization, and Writing - Original Draft \& Editing.

JCC: Writing - Review \& Editing, Funding Acquisition, Supervision \& Resources, and Project Administration.

\section{FINANCIAL DISCLOSURE}

This research was funded by the Taipei American School. The funders had no role in study design, data collection and analysis, decision to publish, or preparation of the manuscript.

\section{COMPETING INTERESTS}

The authors have declared that no competing interests exist.

[1] Geim, A.K.; Novoselov, K.S. Nat. Mater. 2007, 6 (Mar 01), 183-191.

[2] Papageorgiou, D.; Kinloch, I.A.; Young, R.J. Prog. Mater. Sci. 2017, 90 (Oct), 75-127.

[3] Castro Neto, A.H.; Guinea, F.; Peres, N.M.R.; Novoselov, K.S.; Geim, A.K. Rev. Mod. Phys. 2009, 81 (1), 109.

[4] Wang, S.; Cole, I.S.; Li, Q. RSC Adv. 2016, 6 (Sep 08), 89867-89878.

[5] Chen, W.; Lv, G.; Hu, W.; Li, D.; Chen, S.; Dai, Z. Nanotechnol. Rev. 2018, 7 (2), 157-185.

[6] Zhu, S.; Song, Y.; Wang, J.; Wan, H.; Zhang, Y.; Ning, Y.; Yang, B. Nano Today 2017, 13 (Apr), 10-14.

[7] Diao, S.; Zhang, X.; Shao, Z.; Ding, K.; Jie, J.; Zhang, X. Nano Energy 2017, 31 (Jan), 359-366.

[8] Guo, J.; Zhu, H.; Sun, Y.; Tang, L.; Zhang, X. J. Mater. Chem. A 2016, 4 (13), 4783-4789.

[9] Zhang, R.; Ding, Z. Journal of Analysis and Testing 2018, 2 (1), 45-60.

[10]Zhao, S.; Lavie, J.; Rondin, L.; Orcin-Chaix, L.; Diederichs, C.; Roussignol, P.; Chassagneux, Y.; Voisin, C.; Müllen, K.; Narita, A.; Campidelli, S.; Lauret, J.S. Nat. Commun. 2018, 9 (1).

[11] Ain, N.; Eriksson, M.O.; Schmidt, S.; Asghar, M.; Lin, P.C.; Holtz, P.O.; Syväjärvi, M.; Yazdi, G.R. Nanomaterials 2016, 6 (11), 198.

[12] Kumar, G.S.; Roy, R.; Sen, D.; Ghorai, U.K.; Thapa, R.; Mazumder, N.; Saha, S.; Chattopadhyay, K.K. Nanoscale 2014, 6 (Dec 05), 3384-3391.

[13] Li, Y.; Shu, H.; Niu, X.; Wang, J. J. Phys. Chem. C 2015, 119 (44), 24950-24957.

[14] Ahirwar, S.; Mallick, S.; Bahadur, D. ACS Omega 20172 (11), 8343-8353. 
[15] Sudesh; Kumar, N.; Das, S.; Bernhard, C.; Varma, G.D. Supercond. Sci. Technol. 2013, 26 (9). (Figure 1)

[16] William E. Wallace, "Infrared Spectra" in NIST Chemistry WebBook, NIST Standard Reference Database Number 69, Eds. P.J. Linstrom and W.G. Mallard, National Institute of Standards and Technology, Gaithersburg MD, 20899, https://doi.org/10.18434/T4D303, (retrieved July 17, 2019).

[17]Bergantini, A.; Pilling, S.; Rothard, H.; Boduch, P.; Andrade, D.P.P. Mon. Not. R. Astron. Soc. 2014, 437 (3). (Figure 1)

[18] Wishart, D.S.; Knox, C.; Guo, A.C.; Eisner, R.; Young, N.; Gautam, B.; Hau, D.D.; Psychogios, N; Dong, E.; Bouatra, S.; Mandal, R.; Sinelnikov, I.; Xia, J.; Jia, L.; Cruz, J.A.; Lim, E.; Sobsey, C.A.; Shrivastava, S.; Huang, P.; Liu, P.; Fang, L.; Peng, J.; Fradette, R.; Cheng, D.; Tzur, D.; Clements, M.; Lewis, A.; De Souza, A.; Zuniga, A.; Dawe, M.; Xiong, Y.; Clive, D.; Greiner, R.; Nazyrova, A.; Shaykhutdinov, R.; Li, L.; Vogel, H.J.; Forsythe, I. Nucleic Acids Res. 2009, 37 (Database Issue).

[19] Song, L.; Shi, J.; Lu, J.; Lu, C. Chem. Sci. 2015, 6 (8), 4846-4850. (Figure 4b)

[20] Li, L.; Wu, G.; Yang, G.; Peng, J.; Zhao, J.; Zhu, J.-J. Nanoscale 2013, 5 (Jan 10), 4015-4039. 